\title{
Application-Specific Arithmetic in High-Level Synthesis Tools
}

\author{
YOHANN UGUEN, FLORENT DE DINECHIN, and VICTOR LEZAUD, Univ. Lyon, INSA Lyon, \\ Inria, CITI \\ STEVEN DERRIEN, Univ. Rennes 1, IRISA
}

\begin{abstract}
This work studies hardware-specific optimization opportunities currently unexploited by high-level synthesis compilers. Some of these optimizations are specializations of floating-point operations that respect the usual semantics of the input program without changing the numerical result. Some other optimizations, locally triggered by the programmer thanks to a pragma, assume a different semantics, where floating-point code is interpreted as the specification of computation with real numbers. The compiler is then in charge to ensure an application-level accuracy constraint expressed in the pragma and has the freedom to use non-standard arithmetic hardware when more efficient. These two classes of optimizations are prototyped in the GeCoS source-to-source compiler and evaluated on the Polybench and EEMBC benchmark suites. Latency is reduced by up to $93 \%$, and resource usage is reduced by up to $58 \%$.
\end{abstract}

CCS Concepts: • Computer systems organization $\rightarrow$ High-level language architectures; Hardware $\rightarrow$ Arithmetic and datapath circuits; Reconfigurable logic applications; Hardware description languages and compilation;

Additional Key Words and Phrases: High-level synthesis, computer arithmetic, floating point, operator specialization

\section{ACM Reference format:}

Yohann Uguen, Florent de Dinechin, Victor Lezaud, and Steven Derrien. 2020. Application-Specific Arithmetic in High-Level Synthesis Tools. ACM Trans. Archit. Code Optim. 17, 1, Article 5 (March 2020), 23 pages.

https://doi.org/10.1145/3377403

\section{INTRODUCTION}

Many case studies have demonstrated the potential of field-programmable gate arrays (FPGAs) as accelerators for a wide range of applications, from scientific and financial computing to signal and data processing, bioinformatics, molecular dynamics, stencil computations and cryptography [46]. FPGAs offer massive parallelism and programmability at the bit level. These characteristics enable programmers to exploit a range of techniques that avoid many bottlenecks of classical

This work is an extension of a conference paper [43] and proposes other floating-point arithmetic optimizations for highlevel synthesis tools. The previous optimizations were modifying the semantic of the original program to boost performance and accuracy. This work adds semantic preserving optimizations that should be applied in every scenario.

Authors' address: Y. Uguen, F. de Dinechin, and V. Lezaud, CITI, bâtiment Claude Chappe, 6 avenue des Arts, F-69621 Villeurbanne, France; emails: yo.uguen@gmail.com, \{florent.de-dinechin, victor.lezaud\}@insa-lyon.fr; S. Derrien, ISTICIRISA, Campus de Beaulieu, 35042 Rennes Cedex, France; email: steven.derrien@univ-rennes1.fr.

Permission to make digital or hard copies of all or part of this work for personal or classroom use is granted without fee provided that copies are not made or distributed for profit or commercial advantage and that copies bear this notice and the full citation on the first page. Copyrights for components of this work owned by others than the author(s) must be honored. Abstracting with credit is permitted. To copy otherwise, or republish, to post on servers or to redistribute to lists, requires prior specific permission and/or a fee. Request permissions from permissions@acm.org.

(C) 2020 Copyright held by the owner/author(s). Publication rights licensed to ACM.

1544-3566/2020/03-ART5

https://doi.org/10.1145/3377403 
von Neumann computing: dataflow operation without the need of instruction decoding; massive register and memory bandwidth without contention on a register file and single memory bus; and operators and storage elements tailored to the application in nature, number, and size.

However, to unleash this potential, development costs for FPGAs are orders of magnitude higher than classical programming. High performance and high development costs are the two faces of the same coin. One reason for the high development costs on FPGAs is that they inherited their programming model from digital circuit design. The hardware description languages (HDLs) used have a very different programming paradigm than the languages used for software design. When accelerating software code with FPGAs, software-oriented languages such as $\mathrm{C} / \mathrm{C}++$ are increasingly being considered as HDLs. This has many advantages. Experimenting with different options of FPGA acceleration is simpler because no rewriting of software into HDL is needed. The language itself is more widely known than any HDL. The sequential execution model makes designing and debugging much easier. One can use software execution on a processor for simulation. All of this drastically reduces development time.

Compiling a software description (e.g., a C program) into hardware is called high-level synthesis (HLS). Examples of HLS tools include Vivado HLS [3], Intel HLS [2], and Catapult C, ${ }^{1}$ among others [35]. Turning a $\mathrm{C}$ description into a circuit requires extracting parallelism from sequential programs constructs (e.g., loops) and exposing this parallelism in the target design. Today's HLS tools are reasonably good at this task and can automatically synthesize highly efficient pipelined dataflow architectures.

Early HLS tools had a bottom-up approach, inherited from hardware design, of assembling optimized hardware IP components into more complex designs. This approach failed to scale to large and irregular applications. Conversely, modern HLS tools are built upon mainstream compiler projects such as GCC or Clang/LLVM. This top-down approach, from the language down to the hardware, ensures comprehensive language support and a large number of state-of-the-art optimization techniques $[5,32,33]$. However, most of these optimizations were designed for standard CPUs. It is therefore relevant to question if they make sense in an FPGA context [25]. It is also relevant to attempt to identify new optimizations that did not make sense in a software context but make sense in the FPGA context. Even hardware-specific optimizations that were already performed by some of the early HLS tools can be reconsidered and improved in the new context of modern optimizing compiler frameworks.

This is the main objective of the present work, with a focus on arithmetic-related optimizations.

Consider, for example, the integer multiplication by a constant. Optimization of hardware constant multiplication has been the subject of much research [4, 14, 23, 30, 41, 47], some of which is specific to FPGAs $[8,11,48,49]$. Some of the early HLS tools could exploit this literature and generate an optimized architecture. However, it was not optimized for the big picture: this is what modern compiler frameworks provide, with global program transformations such as (interprocedural) constant propagation and folding, as well as strength reduction [33]. Unfortunately, some optimizations of the leaf hardware operators got lost in this transition to modern compiler frameworks, as we now show.

Listing 1 implements a simple integer multiplication by 7. Listing 2 shows the assembly code of Listing 1 when compiled with gcc 7.4.0 without any particular optimization flag, targeting an x86 microprocessor. One can see that the multiplication by 7 has been transformed by the compiler into a shift-and-add algorithm (here actually a shift-and-sub, hardware addition and subtraction being equivalent in this context): $7 x=8 x-x=2^{3} x-x$, where the multiplication by $2^{3}$ is a simple shift left by 3 bits. Both on GCC or Clang/LLVM, if the code is compiled using a -O2 optimization

\footnotetext{
${ }^{1}$ Catapult C Synthesis, Mentor Graphics, 2011: http://calypto.com/en/products/catapult/overview/.
} 


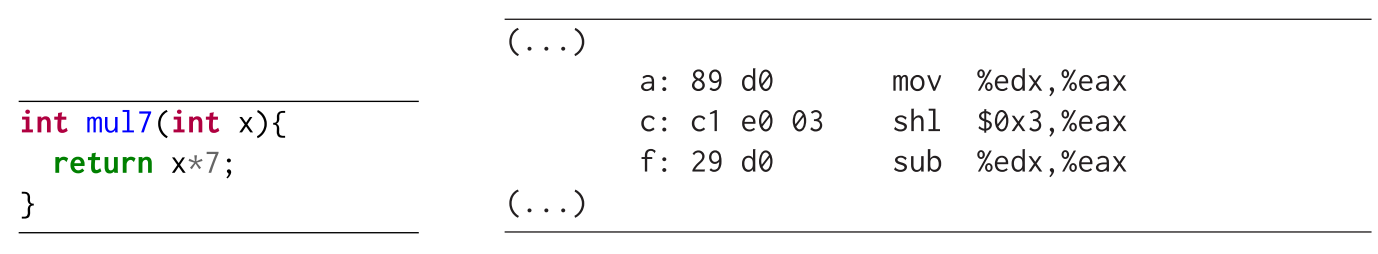

Listing 1. C code.

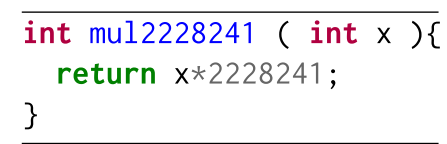

Listing 3. C code.
Listing 2. Objdump of Listing 1 when compiled with gcc (x86).

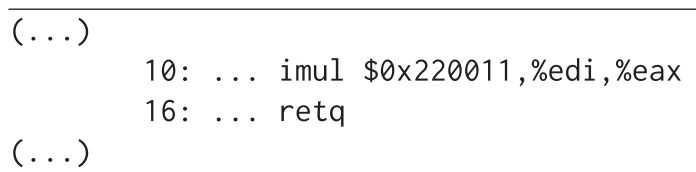

Listing 4. Objdump of Listing 3 compiled with Clang/LLVM -O2 (x86).

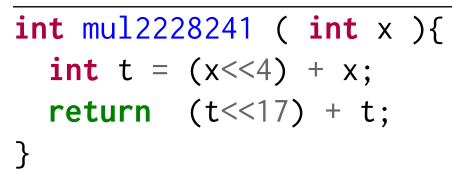

Listing 5. C code using a shift-and-add algorithm.

flag, the algorithm is the same but the multiplication by 8 is implemented by the lea instruction in a slightly less obvious way.

As a consequence, the architecture produced by an HLS tool based on GCC or Clang/LLVM will use a shift-and-add algorithm. It makes even more sense in HLS, since the constant shifts reduce to wires and therefore cost very little hardware. Indeed, the synthesis of Listing 1 in VivadoHLS reports 32 look-up tables (LUTs) (on a Kintex7 FPGA), the cost of one 32-bit adder. Experiments with Vivado HLS (based on Clang/LLVM) and Intel HLS (based on GCC) show that for all constant multiplications that can be implemented as an addition, these tools instantiate an adder instead of a multiplier.

Now consider the multiplication by another constant in Listing 3. In this example, the Clang/LLVM x86 back-end keeps the operation as a multiplication.

Indeed, the synthesis of this operator by VivadoHLS on a Kintex reports 2 LUTs and 2 DSPs, which are the resources needed to implement a 32-bit multiplier.

However, although the constant looks more complex, it barely is: the multiplication by 2228241 can be implemented in two additions as $2228241=2^{17} \cdot 17+17$ : first compute $t=17 x=2^{4} x+x$ (one addition), then compute $2228241 x=2{ }^{17} t+t$ (another addition). Still, neither the $\mathrm{x} 86$ back-end of Clang/LLVM nor GCC use a shift-and-add in this case. The rationale could be the following: the cost of one addition will always be lower than or equal to the cost of a multiplication, whatever the processor, so replacing one multiplication with one addition is always a win. Conversely, it may happen on some (if not most) processors that the cost of two additions and two shifts is higher than the cost of one multiplication.

Is this true in an HLS context? The best architecture for this multiplication, achieved by the C program of Listing 5, consists of two adders: one that computes the 32 lower bits of $t=17 x=$ $2^{4} x+x$ (and should cost only 28 LUTs, since the lower 4 bits are those of $x$ ) and one that computes 


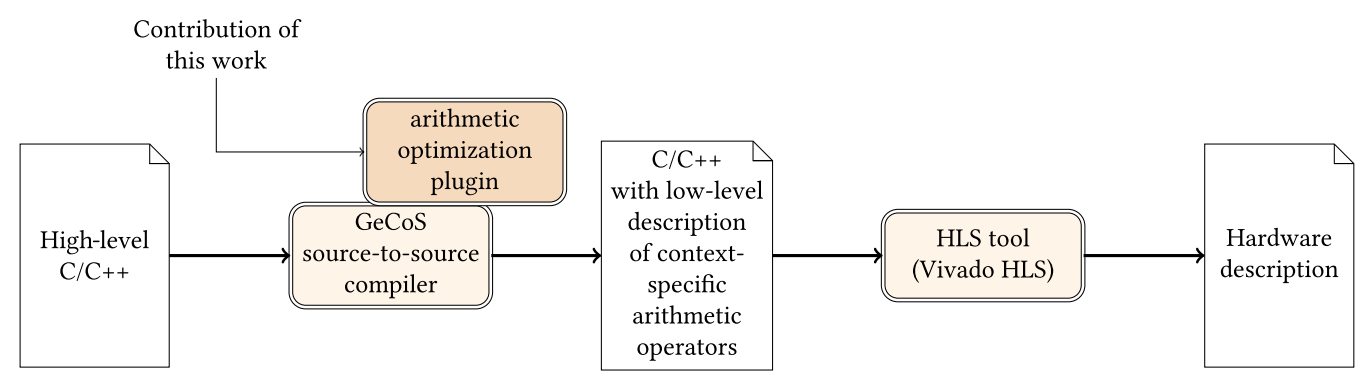

Fig. 1. The proposed compilation flow.

the 32 lower bits of $2^{17} t+t$ (and should cost $32-17=15$ LUTs for the same reason; the 17 lower bits are those of $t$ ). The total cost should be 43 LUTs.

For Listing 5, VivadoHLS indeed reports 46 LUTs, very close to the predicted 43 (and not much higher than the cost of the multiplication by 7 ).

In summary, the observation is that the arithmetic optimization has been completely delegated to the underlying compiler x86 back-end, and that we have a case here for enabling further optimizations when targeting hardware or FPGAs.

The broader objective of the present work is to list similar opportunities of hardware-specific arithmetic optimizations that are currently unexploited and demonstrate their effectiveness. We classify these optimizations in two broad classes:

- Optimizations that strictly respect the semantic of the original program are presented in Section 2. The previous constant multiplication examples belong to this class; we also discuss division by a constant, and we add in this section a few floating-point optimizations that make sense only in a hardware context. This section should be perfectly uncontroversial: all optimizations in this class should be available in an HLS flow as soon as they improve some metric of performance. The only reason it is not yet the case is that commercial HLS tools are relatively young and do not include the portfolio of HLS optimizations that have been researched.

- Optimizations that relax (and we argue, only for the better) the constraint of preserving the program semantics are presented in Section 3. In this more controversial and forward-looking section, we assume that programmers who used floating-point datatypes in their programs intended to compute with real numbers, and we consider optimizations that lead to cheaper and faster but also more accurate hardware. This approach is demonstrated in depth in examples involving floating-point summations and sums of products.

In each case, we use a compilation flow illustrated in Figure 1 that involves one or several source-to-source transformations using the GeCoS framework [19] to improve the generated designs. Source-to-source compilers are very convenient in an HLS context, as they can be used as optimization front-ends on top of closed-source commercial tools. This approach is not new, as source-to-source compilers are already used in an HLS context for dataflow optimization [9].

Finally, Section 4 concludes and discusses to what we believe HLS tools should evolve.

\section{OPTIMIZATION EXAMPLES THAT DO NOT CHANGE THE PROGRAM SEMANTIC}

The arithmetic optimizations that fit in this section go well beyond the constant multiplications studied in Section 1. In particular, there are opportunities of floating-point optimizations in FPGAs that are more subtle than operator specialization. 


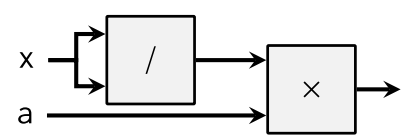

(a) Original circuit

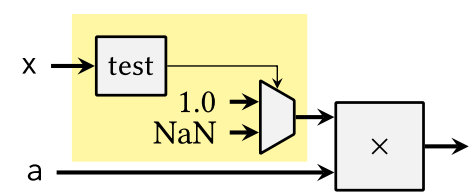

(b) Divider specialization

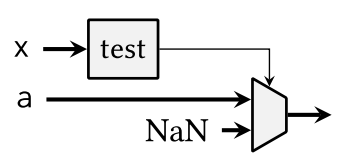

(c) Multiplier specialization

Fig. 2. Optimization opportunities for floating-point $x / x * a$.

\subsection{Floating-Point Corner-Case Optimization}

Computing systems follow the IEEE-754 standard [1] on floating-point arithmetic, which was introduced to normalize computations across different CPUs. Based on this standard, the C standard prevents compilers from performing some floating-point optimizations. Here are a some examples that can be found in the C11 standard [26]:

- $x / x$ and 1.0 are not equivalent if $x$ is zero, infinite, or $\mathrm{NaN}$ (in which case, the value of $x / x$ is $\mathrm{NaN}$ ).

- $x-y$ and $-(y-x)$ are not equivalent because $1.0-1.0$ is +0 but $-(1.0-1.0)$ is -0 (in the default rounding direction).

- $x-x$ and 0 are not equivalent if $x$ is $\mathrm{NaN}$ or infinite.

- $0 \times x$ and 0 are not equivalent if $x$ is NaN, infinite, or -0 .

- $x+0$ and $x$ are not equivalent if $x$ is -0 , because $(-0)+(+0)$, in the default rounding mode (to the nearest), yields +0 , not -0 .

- $0-x$ and $-x$ are not equivalent if $x$ is +0 , because $-(+0)$ yields -0 , but $0-(+0)$ yields +0 .

Of course, programmers usually do not write $x / x$ or $x+0$ in their code. However, other optimization steps, such as code hoisting, or procedure specialization and cloning, may lead to such situations: their optimization is therefore relevant in the context of a global optimizing compiler [33].

Let us consider the first example (the others are similar). A compiler is not allowed to replace $x / x$ with 1.0 unless it is able to prove that $x$ will never be zero, infinity, or NaN. This is true for HLS, as well as for a standard compiler. However, it could replace $x / x$ with something like (is_zero $(x)||$ is_infty $(x)||$ is_nan $(x)$ )?NaN:1.0; . This is, to our knowledge, not implemented. The reason is again probably that in software, the test on $x$ becomes more expensive than the division.

However, if implemented in hardware, this test is quite cheap: it consists of detecting if the exponent bits are all zeroes (which capture the 0 case) or all ones (which captures both infinity and $\mathrm{NaN}$ cases). The exponent is only 8 bits for single precision and 11 bits for double precision.

In an FPGA context, it therefore makes perfect sense to replace $x / x$ (Figure 2(a)) with an extremely specialized divider depicted in Figure 2(b). Furthermore, the two possible values are interesting to propagate further (1.0 because it is absorbed by multiplication, NaN because it is extremely contagious). Therefore, this optimization step enables further ones, where the multiplexer is pushed down the computation, as illustrated by Figure 2(c).

Note that Figure 2(c) replaces $1.0 * a$ by $a$ : this is a valid floating-point optimization in the sense that it is valid even if $a$ is a signed zero, infinity, or NaN.

Occurrences of $x-x, 0 \times x, x+0,0-x$ can similarly be replaced with a multiplexer and very little logic, and may similarly enable further optimizations.

Since these arithmetic optimizations are expected to be triggered by optimizations (procedure specialization) and trigger further optimizations (conditional constant propagation), they need to be implemented and evaluated within an optimizing compiler. The source-to-source flow depicted 


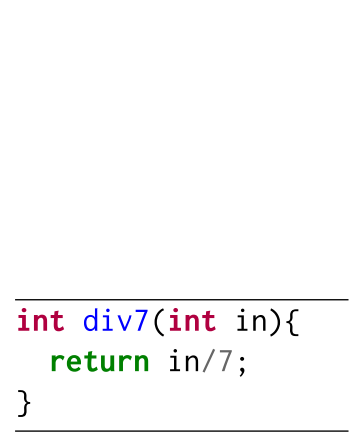

Listing 6. C code.

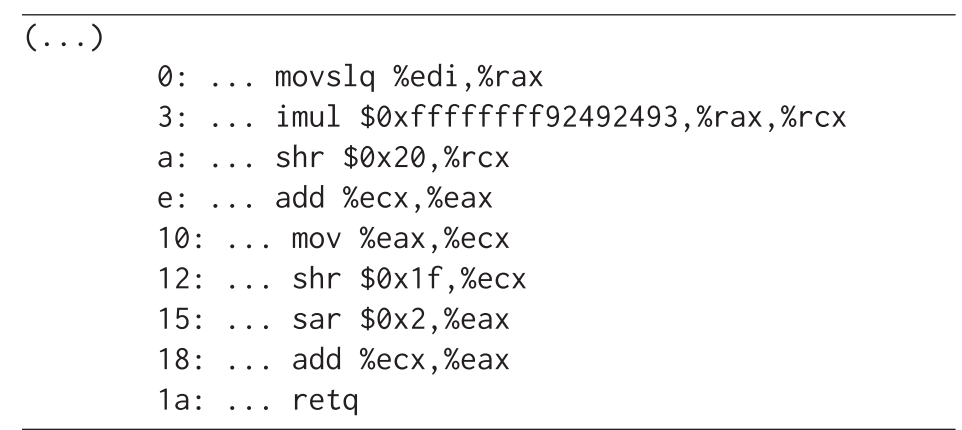

Listing 7. Objdump of Listing 6 when compiled with Clang -O2 (x86).

in Figure 1 is ill suited to studying such cascaded optimizations. Furthermore, the multiple conditional constant propagation that transforms Figure 2(b) into Figure 2(c) is probably not implemented yet, as it does not make much sense in software. This evaluation is therefore left outside the scope of the present article.

In the following, we focus on FPGA-specific semantic-preserving optimizations that do not trigger further optimizations.

\subsection{Integer Multiplication by a Constant}

Multiplication by a constant was mentioned in Section 1 . We just refer to the rich existing literature on the subject $[4,8,11,14,23,30,41,47-49]$. These are mostly academic works, but back-end tools already embed some of it, so this optimization could be the first to be implemented. An issue is that its relevance, in the big picture of a complete application, is not trivial: replacing DSP resources with logic resources is an optimization only in a design that is more DSP intensive than logic intensive. In addition, as soon as a logic-based constant multiplier requires more than a handful of additions, it may entail more pressure on the routing resources as well. Discussing this trade-off in detail in the context of an application is outside the scope of the present article.

\subsection{Integer Division by a Small Constant}

Integer division by a constant adds one more layer of optimization opportunities. In some cases, as illustrated by Listing 6 and Listing 7, a compiler is able to transform this division into a multiplication by a (suitably rounded) reciprocal. This then triggers the previous optimization of a constant multiplier. Actually, one may observe that on this example, the constant $1 / 7$ has the periodic pattern $100100100100100100100100100_{2}$ (hidden in the hexadecimal pattern $924_{16}$ in Listing 7). This enables a specific optimization of the shift-and-add constant multiplication algorithm [10].

Table 1 shows synthesis results on the two FPGA mainstream HLS tools at the time of this writing (Vivado HLS and Intel HLS). As a reminder of the resource names used by these tools, LUTs and ALMs are look-up tables, Regs. and FFs are registers, DSPs are dedicated hardware blocks (essentially for multipliers), SRLs are shift registers, and MLABs are aggregated LUTs to emulate small RAM blocks. The timing constraint was set to $100 \mathrm{MHz}$; however, this factor is not important here, as it does not change the structure of the generated operators. The goal is to observe the optimizations performed (or not) by the tools. Here is what we can infer from this table:

- The generic divider (Divisor=x) is based on Xilinx on a shift-and-add algorithm, whereas on Intel a polynomial approach is used [36] that consumes multipliers and DSP resources.

- Both tools correctly optimize the division by a power of 2 , converting it into a shift. 
Table 1. Synthesis Results for the Division $y / x$, Where $y$ is a 32-Bit Integer Variable and $x$ Is Either a 32-Bit Integer Variable or an Integer Constant between 1 and 9

(a) Vivado HLS

\begin{tabular}{ccccc}
\hline Divisor & LUTs & Regs. & DSPs \\
\cline { 1 - 2 } \cline { 3 - 5 } & & 217 & 294 & 0 \\
1 & & 0 & 0 & 0 \\
2 & & 78 & 0 & 0 \\
3 & & 162 & 67 & 4 \\
4 & & 77 & 0 & 0 \\
5 & & 162 & 67 & 4 \\
6 & & 162 & 67 & 4 \\
7 & & 163 & 68 & 4 \\
8 & & 75 & 0 & 0 \\
9 & & 160 & 65 & 4 \\
\hline
\end{tabular}

(b) Intel HLS

\begin{tabular}{ccccc}
\hline ALMs & FFs & RAMs & DSPs & MLABs \\
\hline 668 & 707 & 4 & 10 & 9 \\
26.5 & 45 & 0 & 0 & 0 \\
31.5 & 41 & 0 & 0 & 0 \\
126.5 & 102 & 0 & 0 & 2 \\
30.5 & 40 & 0 & 0 & 0 \\
132.5 & 110 & 0 & 0 & 2 \\
123.5 & 99 & 0 & 0 & 2 \\
136 & 106 & 0 & 0 & 2 \\
30.5 & 40 & 0 & 0 & 0 \\
165.5 & 103 & 0 & 0 & 2 \\
\hline
\end{tabular}

Synthesis is performed using Vivado HLS 2019.1 for Kintex 7, and Intel HLS 19.2 for Arria 10, targeting $100 \mathrm{MHz}$.

\begin{tabular}{|r|r}
\hline 756 & 3 \\
\hline 17 & 258 \\
\hline 17 & 7 \\
\hline 26 & \\
\hline 5 & \\
2 &
\end{tabular}

We first compute the Euclidean division of 7 by 3 . This gives the first digit of the quotient, here 2 , and the remainder is 1 . In other words $7=3 \times 2+1$. The second step divides 77 by 3 by first rewriting $77=70+7=3 \times 20+10+7$ : dividing 17 by 3 gives $17=3 \times 5+2$. The third steps rewrites $776=770+6=250+20+6$ where $26=3 \times 8+2$, hence $776=3 \times 258+2$.

The only computation in each step is the Euclidean division by 3 of of a number between 0 and 29: it can be pre-computed for these 30 cases and stored in a look-up table (LUT).

Fig. 3. Illustrative example: division by 3 in decimals.

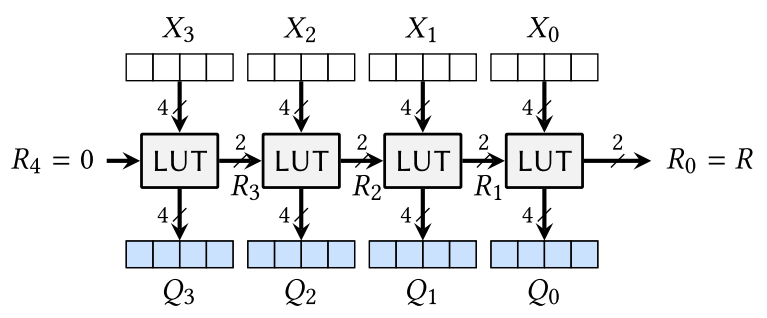

Fig. 4. Architecture for division by 3 of a 16-bit number written in hexadecimals, using LUTs with 6 input bits.

- Division by non-power of 2 integers is implemented by a multiplication by the inverse on Xilinx (it consumes DSP blocks). On Intel, this multiplication is further optimized as a logiconly operation.

For the division of an integer by a very small constant, the best alternative is the algorithm described by Ugurdag et al. [45]. It is based on the decimal paper-and-pencil algorithm illustrated in Figure 3. Figure 4 describes an unrolled architecture for a binary-friendly variant of this algorithm. There, the input $X$ is written in hexadecimal (each 4-bit word $X_{i}$ is an hexadecimal digit). The quotient bits come out in hexadecimal. The remainder of the division by 3 is always between 0 and 2, and therefore fits on 2 bits. Each look-up table (LUT) on the figure thus stores the quotient $Q_{i}$ and the remainder $R_{i}$ of the division by 3 of a number $R_{i+1} X_{i}$. This number is between $00_{h}$ 
Table 2. Synthesis Results for the Division $y / x$, Where $y$ Is a 64-Bit Integer Variable and $x$ is Either a 64-Bit Integer Variable or a Constant between 1 and 9

(a) C Division

\begin{tabular}{|c|c|c|c|c|c|}
\hline Divisor & LUTs & Reg. & DSPs & Cycles @ Freq & $\overline{\text { WCT }}$ \\
\hline$x$ & 8704 & 8588 & 0 & $67 @ 291 \mathrm{MHz}$ & 230.24 \\
\hline 2 & 162 & 193 & 0 & $2 @ 464 \mathrm{MHz}$ & 4.31 \\
\hline 3 & 550 & 728 & 16 & $22 @ 343 \mathrm{MHz}$ & 64.13 \\
\hline 4 & 161 & 190 & 0 & $2 @ 453 \mathrm{MHz}$ & 4.41 \\
\hline 5 & 548 & 726 & 16 & $22 @ 348 \mathrm{MHz}$ & 63.21 \\
\hline 6 & 548 & 726 & 16 & $22 @ 348 \mathrm{MHz}$ & 63.21 \\
\hline 7 & 548 & 726 & 16 & $22 @ 348 \mathrm{MHz}$ & 63.21 \\
\hline 8 & 159 & 187 & 0 & 2 @ $451 \mathrm{MHz}$ & 4.43 \\
\hline 9 & 550 & 726 & 16 & $22 @ 351 \mathrm{MHz}$ & 62.67 \\
\hline
\end{tabular}

(b) Ugurdag et al. [45] Division in HLS

\begin{tabular}{crrr}
\hline LUTs & Reg. & Cycles @ Freq & WCT \\
\hline NA & NA & NA & NA \\
163 & 193 & $2 @ 396 \mathrm{MHz}$ & 5.05 \\
301 & 230 & $16 @ 364 \mathrm{MHz}$ & 43.95 \\
162 & 190 & $2 @ 406 \mathrm{MHz}$ & 4.92 \\
391 & 272 & $22 @ 316 \mathrm{MHz}$ & 69.62 \\
222 & 208 & $16 @ 362 \mathrm{MHz}$ & 44.19 \\
414 & 469 & $22 @ 316 \mathrm{MHz}$ & 69.62 \\
160 & 187 & $2 @ 390 \mathrm{MHz}$ & 5.12 \\
371 & 354 & $32 @ 294 \mathrm{MHz}$ & 108.84 \\
\hline
\end{tabular}

Synthesis is performed using Vivado HLS 2019.1 for Kintex 7, and Intel HLS 19.2 for Arria 10, targeting $100 \mathrm{MHz}$.

and $2 F_{h}$. On a recent LUT-based FPGA, each 6-input, 6-output LUT of Figure 4 consumes exactly 6 FPGA LUTs: This architecture is very well suited to FPGAs.

Table 2 compares the performance on Xilinx of the division of a 64-bit integer by a small constant, when left to the Vivado HLS tool (left part), and when first replaced by an HLS description of the architecture of Figure 4 by a source-to-source transformation (right part of the table). For constants smaller than 9, all of the metrics (logic resources, DSP, latency, and frequency) are improved by this transformation. The wall clock time (WCT) is also reported. Throughout the rest of this article, the WCT is given in nanoseconds, unless stated otherwise. As the constant grows larger, the latency degrades and the resource consumption increases: for division by 9 , we already have a worst latency and frequency than the default multiplication-based implementation, but still with much less resources. For smaller constants, the transformed operators reduce the resource usage by at least a factor of 2 while still requiring a smaller or equal WCT.

\subsection{Floating-Point Multiplications and Division by Small Constants}

As illustrated in Table 3, even fewer optimizations are implemented for floating-point multiplications and divisions by a constant:

- Both Vivado HLS and Intel HLS are able to remove the constant multiplication and division by 1.0 (unsurprisingly, since it is a valid simplification in software compilers).

- Intel HLS seems to optimize constant multiplications (it never requires a DSP). Vivado HLS, however, does not even optimize floating-point multiplications by 2.0 or a power of 2 . This class of operations should resume to an addition on the exponents and specific overflow/ underflow logic.

- Both tools use a specific optimization when dividing by a power of 2 . This can easily be explained by looking at the assembly code generated by GCC or Clang/LLVM in such cases: both compilers will transform a division by 4.0 into a multiplication by 0.25 , which is bitfor-bit equivalent and much faster on most processors.

- Both tools use a standard divider for constants that are not a power of 2, with minor resource reductions thanks to the logic optimizer.

Again we may question the relevance of these choices on FPGAs. It is indeed possible to design floating-point versions of both constant multiplications [6] and constant divisions [45] that are bit-for-bit compatible with IEEE correctly rounded ones. For instance, in the case of division, 
Table 3. Synthesis Results for the Multiplication $y * x$ and the Division $y / x$, Where $y$ Is a Single-Precision Floating-Point Variable and $x$ Is Either a Single-Precision Floating-Point Variable or an Integer Constant between 1 and 5

(a) Vivado HLS

\begin{tabular}{|c|c|c|c|c|c|}
\hline \multirow{7}{*}{$\stackrel{\Xi}{\Xi}$} & Value & LUTs & Regs. & DSPs & SRLs \\
\hline & $x$ & $\overline{74}$ & 99 & 3 & 0 \\
\hline & 1.0 & 0 & 0 & 0 & 0 \\
\hline & 2.0 & 53 & 67 & 3 & 0 \\
\hline & 3.0 & 53 & 67 & 3 & 0 \\
\hline & 4.0 & 59 & 67 & 3 & 0 \\
\hline & 5.0 & 59 & 67 & 3 & 0 \\
\hline \multirow{6}{*}{ Div } & $x$ & 754 & 391 & 0 & 24 \\
\hline & 1.0 & 0 & 0 & 0 & 0 \\
\hline & 2.0 & 58 & 67 & 3 & 0 \\
\hline & 3.0 & 686 & 244 & 0 & 23 \\
\hline & 4.0 & 58 & 67 & 3 & 0 \\
\hline & 5.0 & 688 & 244 & 0 & 24 \\
\hline
\end{tabular}

(b) Intel HLS

\begin{tabular}{ccccc}
\hline ALMs & FFs & RAMs & DSPs & MLABs \\
\hline 76.5 & 104 & 0 & 1 & 2 \\
26.5 & 45 & 0 & 0 & 0 \\
85.5 & 63 & 0 & 0 & 2 \\
111 & 62 & 0 & 0 & 2 \\
86 & 60 & 0 & 0 & 2 \\
124 & 61 & 0 & 0 & 2 \\
\hline 343.5 & 706 & 3 & 4 & 7 \\
26.5 & 45 & 0 & 0 & 0 \\
85 & 60 & 0 & 0 & 2 \\
341 & 547 & 3 & 4 & 9 \\
86.5 & 62 & 0 & 0 & 2 \\
331 & 540 & 3 & 4 & 9 \\
\hline
\end{tabular}

Synthesis is performed using Vivado HLS 2019.1 for Kintex 7, and Intel HLS 19.2 for Arria 10, targeting 100 $\mathrm{MHz}$.

Table 4. Synthesis Results of the Division of $y / x$, Where $y$ Is a Floating-Point Number and $x$ Is Either a Floating-Point Variable or an Integer Constant between 1 and 5

(a) Float

\begin{tabular}{|c|c|c|c|c|}
\hline$\overline{\text { Divisor }}$ & LUTs & Reg. & Cycles @ Freq. & $\overline{\text { WCT (ns) }}$ \\
\hline$x$ & 808 & 1444 & $29 @ 451 \mathrm{MHz}$ & 64.30 \\
\hline 2.0 & 63 & 61 & 2 @ $619 \mathrm{MHz}$ & 3.23 \\
\hline 3.0 & 266 & 177 & $10 @ 359 \mathrm{MHz}$ & 27.85 \\
\hline 4.0 & 89 & 62 & 2 @ $608 \mathrm{MHz}$ & 3.28 \\
\hline 5.0 & 292 & 199 & $12 @ 356 \mathrm{MHz}$ & 33.70 \\
\hline 6.0 & 276 & 178 & $10 @ 361 \mathrm{MHz}$ & 27.70 \\
\hline 7.0 & 301 & 193 & $17 @ 360 \mathrm{MHz}$ & 47.22 \\
\hline 8.0 & 85 & 61 & 2 @ $579 \mathrm{MHz}$ & 3.45 \\
\hline 9.0 & 329 & 258 & $17 @ 242 \mathrm{MHz}$ & 70.24 \\
\hline 10.0 & 301 & 198 & $13 @ 325 \mathrm{MHz}$ & 40.00 \\
\hline 11.0 & 328 & 269 & $17 @ 229 \mathrm{MHz}$ & 74.23 \\
\hline
\end{tabular}

(b) Double

\begin{tabular}{ccrr}
\hline LUTs & Reg. & Cycles @ Freq. & WCT (ns) \\
\hline 3266 & 3177 & $30 @ 183 \mathrm{MHz}$ & 163.93 \\
126 & 227 & 3 @ $574 \mathrm{MHz}$ & 5.22 \\
588 & 459 & $17 @ 187 \mathrm{MHz}$ & 90.90 \\
176 & 281 & $3 @ 452 \mathrm{MHz}$ & 6.63 \\
687 & 488 & $22 @ 204 \mathrm{MHz}$ & 107.84 \\
596 & 438 & $17 @ 189 \mathrm{MHz}$ & 89.94 \\
685 & 540 & $22 @ 185 \mathrm{MHz}$ & 118.91 \\
176 & 228 & $3 @ 577 \mathrm{MHz}$ & 5.19 \\
698 & 590 & $32 @ 209 \mathrm{MHz}$ & 153.11 \\
696 & 472 & $22 @ 190 \mathrm{MHz}$ & 115.78 \\
697 & 572 & $32 @ 189 \mathrm{MHz}$ & 169.31 \\
\hline
\end{tabular}

In the constant case, the architecture from Ugurdag et al. [45] is used. Synthesis is performed using Vivado HLS 2019.1 for Kintex 7, and Intel HLS 19.2 for Arria 10, targeting $100 \mathrm{MHz}$.

the remainder $R$ that is output by Figure 4 can be used to determine the proper rounding of the significand quotient (for the full details, see Ugurdag et al. [45]).

As we expect constant multiplications to be properly supported soon (it seems to be already the case on Intel HLS), we focus our evaluation on constant division. Table 4 provides synthesis results of Vivado HLS C++ generated operators for floating-point divisions by small constants. The standard floating-point division is also given for comparison purposes, since Table 3 shows that it is the default architecture. All of these operators can be more/less deeply pipelined to achieve higher/lower frequencies at the expense of latency and registers: we attempt to achieve a frequency comparable to that of the standard divider. 
Table 5. Benchmark Synthesis Results Using Vivado HLS 2019.1 for Kintex 7, Before and After Constant Multiplication and Division Transformations

\begin{tabular}{ccccccrr}
\hline Benchmark & & Type & LUTs & Regs. & DSPs & Cycles @ Freq. & WCT $(\mathrm{ms})$ \\
\cline { 1 - 6 } Fdtd-2d & & Original & 4780 & 6162 & 17 & $153 \mathrm{G} @ 349 \mathrm{MHz}$ & 438.39 \\
& & Transformed & 3326 & 4386 & 17 & $11 \mathrm{G} @ 349 \mathrm{MHz}$ & 31.51 \\
\multirow{2}{*}{ Heat-3d } & Original & 3842 & 6081 & 31 & $193 \mathrm{G} @ 349 \mathrm{MHz}$ & 553.00 \\
& Transformed & 5737 & 6629 & 17 & $141 \mathrm{G} @ 348 \mathrm{MHz}$ & 405.17 \\
\multirow{2}{*}{ Jacobi-1d } & Original & 4274 & 4964 & 3 & $185 \mathrm{M} @ 348 \mathrm{MHz}$ & 0.53 \\
& Transformed & 2167 & 2792 & 3 & $129 \mathrm{M} @ 358 \mathrm{MHz}$ & 0.36 \\
\multirow{2}{*}{ Seidel-2d } & Original & 4419 & 5126 & 6 & $373 \mathrm{G} @ 351 \mathrm{MHz}$ & 1062.67 \\
& Transformed & 1878 & 2341 & 6 & $321 \mathrm{G} @ 304 \mathrm{MHz}$ & 1053.52 \\
\multirow{2}{*}{ Jacobi-2d } & Original & 4588 & 5462 & 9 & $213 \mathrm{G} @ 347 \mathrm{MHz}$ & 613.83 \\
& Transformed & 2548 & 3294 & 9 & $176 \mathrm{G} @ 335 \mathrm{MHz}$ & 525.37 \\
\hline
\end{tabular}

Each optimized constant divider uses fewer resources (up to 5.55 times) and has a lower latency (up to 2.3 times) for a comparable frequency. When dividing by a power of 2 , the cost of the custom divider is virtually nothing (again it resumes to an operation on the exponents).

\subsection{Evaluation in Context}

We implemented a C-to-C source-to-source transformation that detects floating-point multiplications and divisions by constants in the source code and replaces it by a custom operator that is bit-for-bit equivalent. This transformation was implemented as a plug-in within the open source source-to-source GeCoS compiler framework [19], as per Figure 1.

This work was then evaluated on the Polybench benchmark suite [37]. It contains several C programs that fit the polyhedral model. The focus here is on the stencil codes of this benchmark suite. Most of them contain a division by a small constant. Indeed, out of the six stencil codes, five were well suited for our transformations. The Jacobi-1d benchmark contains two divisions by 3 ; Jacobi- $2 \mathrm{~d}$ contains two divisions by 5 ; Seidel-2d contains a division by 9 ; Fdtd- $2 \mathrm{~d}$ contains two divisions by 2 and a multiplication by 0.7 ; finally, Heat-3d contains six divisions by 8 and six multiplications by 2 .

Table 5 compares the synthesis results obtained

- using the original $\mathrm{C}$ code, targeting the maximum frequency achievable, and

- using the code after transformation by our GeCoS plug-in.

Each benchmark benefits from the transformations. WCT is always improved, with latency improved up to 13.9 times for similar frequencies. The benefits of the transformations in terms of timing differ from one benchmark to another, with the best improvements being achieved when the transformed operator is in the critical path of an inner loop.

Concerning resource usage, only the Heat-3d benchmark has more LUTs and registers in the transformed version. However, it has fewer DSPs. This is explained by the transformation of six dividers and two multipliers from DSP-based architectures to LUT-based ones. In all other cases, LUT and register usage is reduced for the same amount of DSPs.

\section{OPTIMIZATION EXAMPLES THAT CHANGE THE PROGRAM SEMANTIC}

From a compiler point of view, the previous transformations were straightforward and semantic preserving. Conversely, the case study in this section replaces the standard semantics of a ACM Transactions on Architecture and Code Optimization, Vol. 17, No. 1, Article 5. Publication date: March 2020. 
Table 6. Synthesis Results of Different Accumulators Using Vivado HLS for Kintex 7 at $100 \mathrm{MHz}$

\begin{tabular}{|c|c|c|c|c|c|c|}
\hline & \multicolumn{2}{|c|}{ float acc } & \multicolumn{2}{|c|}{ double acc } & \multicolumn{2}{|c|}{ 68-Bit Fixed-Point acc } \\
\hline & Listing 8 & Listing 9 & Listing 8 & Listing 9 & Listing 10 & FloPoCo VHDL \\
\hline LUTs & 387 & 835 & 784 & 1908 & 462 & $375^{*}$ \\
\hline DSPs & 0 & 2 & 0 & 3 & 0 & 0 \\
\hline Latency & $400 \mathrm{~K}$ & $100 \mathrm{~K}$ & $400 \mathrm{~K}$ & $100 \mathrm{~K}$ & $100 \mathrm{~K}$ & $100 \mathrm{~K}$ \\
\hline Accuracy & 20 bits & 20 bits & 24 bits & 24 bits & 24 bits & 24 bits \\
\hline
\end{tabular}

${ }^{*}$ Does not include the control part.

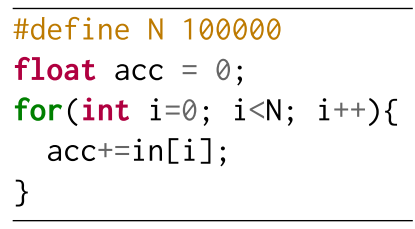

Listing 8. Usual reduction.

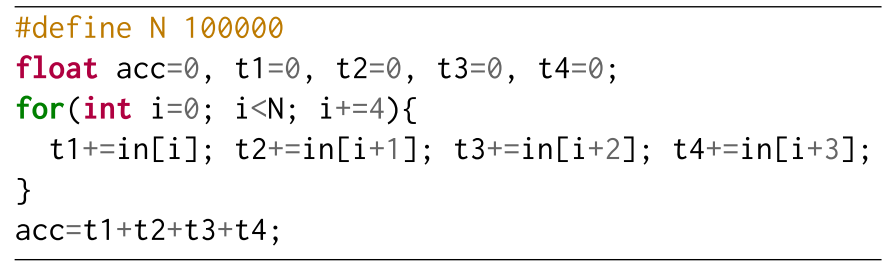

Listing 9. Unrolled reduction to express parallelism.

floating-point code fragment with a new one, based on a user-specified accuracy with respect to the exact computation on the reals. This enables deeper program transformations, such as a change of the internal number representation that enables both improved accuracy and tightened loop-carried dependencies.

Before detailing it, we must digress a little on the subtleties of the management of floating-point arithmetic by compilers.

\subsection{HLS Faithful to the Floats}

Most recent compilers, including the HLS ones [24], attempt to follow established standards, particularly C11, and, for floating-point arithmetic, IEEE-754. This brings the huge advantage of almost bit-exact reproducibility-the hardware will compute exactly the same results as the software. However, it also greatly reduces the freedom of optimization by the compiler. For instance, as floating-point addition is not associative, $\mathrm{C} 11$ mandates that code written $a+b+c+d$ is executed as $((a+b)+c)+d$, although $(a+b)+(c+d)$ would have a shorter latency.

This also prevents exploiting parallelism in floating-point reductions. A reduction is a computation that reduces a set of input values into a reduction location using an associative operation. Examples of reductions include summations, products, and wide logical AND, among others.

Reductions are typically expressed as loops. If floating-point addition were associative, Listing 8 would be an example of reduction, where acc is the reduction location. The first column of Table 6 gives the synthesis results of Listing 8 using Vivado HLS for Kintex7. The latency of a floatingpoint addition is seven cycles in this case. However, using a feedback loop that injects the current denormalized sum as a new input to the adder $[15,18]$, the Vivado HLS IP is able to achieve an initiation interval of four cycles. Still, the adder is only active one cycle out of four due to the loop-carried dependency.

Listing 9 shows a different version of Listing 8 that we manually unrolled in such a way to express more parallelism. However, this transformation assumes that floating-point addition is associative, which it is not. Indeed, Vivado HLS is not allowed to transform Listing 8 into Listing 9. According to $\mathrm{C}$ or $\mathrm{C}++$ semantics, Listing 9 and Listing 8 are not equivalent. Remark that a parallel execution with the sequential semantics is possible but very expensive [28].

Vivado HLS is able to exploit the parallelism in Listing 9 (second column of Table 6). It reports using two floating-point adders. The one inside the loop is now active at each cycle on a different 
sub-sum, hence a factor 4 gain on latency. The second floating-point adder (whose shifts are implemented using DSP blocks) is used for the final additions. Note that Listing 9 represents the easy case when $N$ is a multiple of the initialization interval of the addition: it would become more complex otherwise.

As a final remark, it is important to point out that the accuracy of these solutions is far from perfect: the $100 \mathrm{~K}$ rounding errors in the reduction sum up. For test data, we use the input values in $[i]=(f l o a t) \cos (i)$ as in Muller et al. [34], where $i$ is the input array's index. Therefore, the accumulation computes $\sum_{i}$ in[i]. The golden value to compare with is obtained using the MPFR library [21]. We measure that only 20 bits of the result are correct (out of the 24 bits of a float significand). When computing the sum of the sines, the accuracy is reduced down to 17 bits. A solution to this is to perform the accumulation in double precision (changing the keyword float into double in the second lines of Listings 8 and 9). This is obviously more expensive, as the two middle columns of Table 6 show.

\subsection{Toward HLS Faithful to the Reals}

Many floating-point programmers routinely assume associativity of floating-point addition and multiplication, tweaking their code in the same way as we transformed Listing 8 into Listing 9 , sometimes assisted by source-to-source compilers or "unsafe" compiler optimizations.

The point of view chosen in this work is to go one step further and allow the programmer to express that some of the floating-point $\mathrm{C} / \mathrm{C}++$ program is intended to describe a computation on real numbers. In other words, under programmer control, some of the floats in $\mathrm{C} / \mathrm{C}++$ will be interpreted as real numbers.

This recovers the associativity of the addition, so Listing 8 is again a reduction and can validly be transformed into Listing 9. In addition, this new point of view brings into the picture a new degree of freedom: accuracy. In an hardware context, designers want to tailor the precision (hence the cost) to the accuracy requirements of the application-a classical concern in HLS [7, 22]. Now that $\mathrm{C} / \mathrm{C}++$ describes a computation on the reals, the user should also be able to specify the accuracy of the computation with respect to this exact (real) result. It should then be the task of the compiler to determine the best way to ensure the prescribed accuracy. This is what we explore in the sequel.

\subsection{The Arithmetic Side: Application-Specific Accumulator Support}

Several approaches for performing a floating-point summation using non-standard hardware have been proposed [27, 31]. In this work, we chose an approach better suited to the HLS context: a generalization of an idea developed by Kulisch. He advocated to augment processors with a very large fixed-point accumulator [29] whose 4288 bits would cover the entire range of double-precision floating point and then some more: such an accumulator would remove rounding errors from all possible floating-point additions and sums of products. The added bonus of an exact addition is that it becomes associative, since the loss of associativity in floating point is due to rounding.

So far, Kulisch's full accumulator has proven too costly to appear in mainstream processors. However, in the context of application acceleration with FPGAs, it can be tailored to the accuracy requirements of applications. Its cost then becomes comparable to classical floating-point operators, although it vastly improves accuracy [13]. This operator can be found in the FloPoCo [12] generator and in Intel DSP Builder Advanced. Its core idea, illustrated in Figure 5, is to use a large fixed-point register into which the mantissas of incoming floating-point summands are shifted (top) and then accumulated (middle). A third component (bottom) converts the content of the accumulator back to the floating-point format. The sub-blocks visible on this figure (shifter, adder, and leading zero counter) are essentially the building blocks of a classical floating-point adder. 


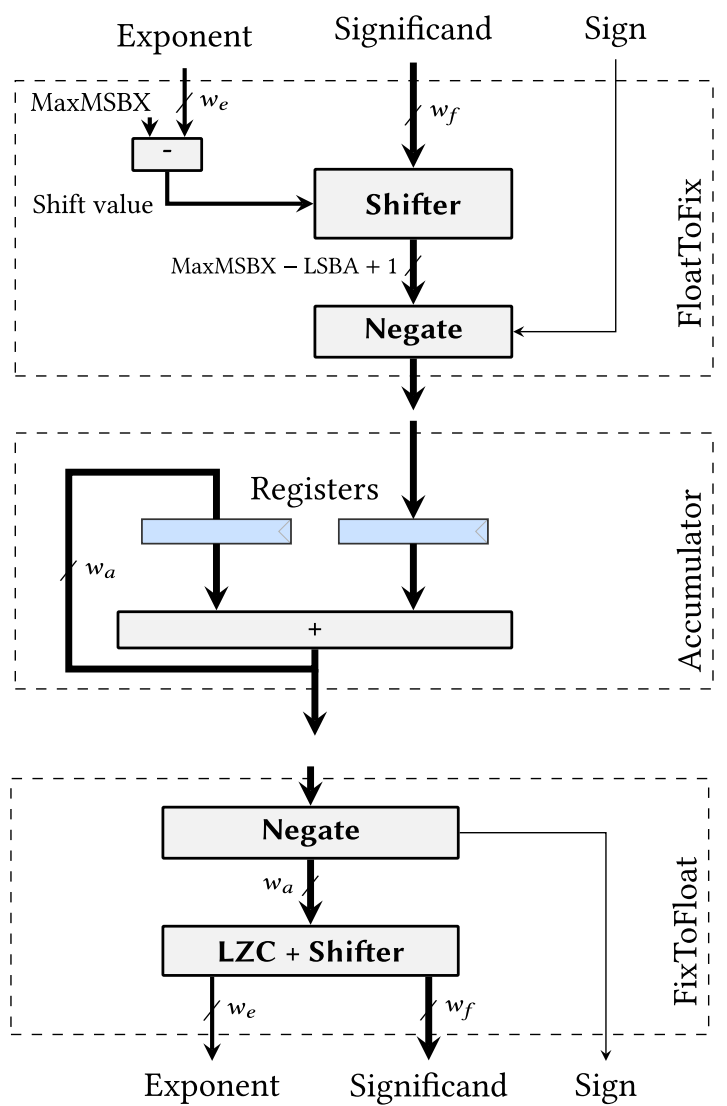

Fig. 5. Conversion from float to fixed point (top), fixed-point accumulation (middle), and conversion from fixed point to float (bottom).

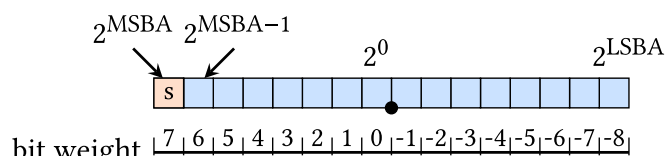

Fig. 6. Fixed-point accumulator format, here $(M S B A, \operatorname{LSBA})=(7,-8)$.

The accumulator used here slightly improves the one offered by FloPoCo [13]:

- It supports subnormal numbers [34].

- In FloPoCo, FloatToFix and Accumulator form a single component, which restricts its application to simple accumulations similar to Listing 8. The decomposition in two components of Figure 5 enable a generalization to arbitrary summations within a loop, as Section 3.4 shows.

3.3.1 The Parameters of a Large Accumulator. The main feature of this approach is that the internal fixed-point format is configurable to control accuracy. For this purpose, this format (represented in Figure 6) has two parameters: 


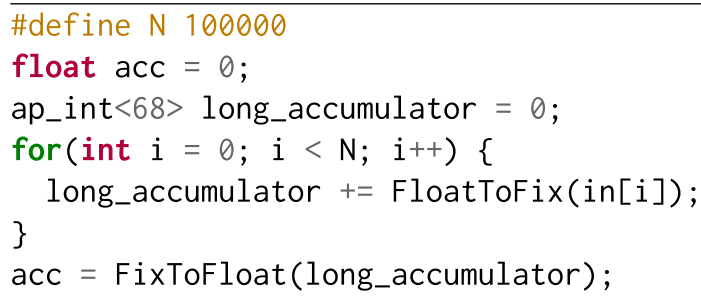

Listing 10. Sum of floats using the large fixed-point accumulator.

- MSBA is the weight of the most significant bit of the accumulator. For example, if MSBA = 17 , the accumulator can accommodate values up to a magnitude of $2^{17} \approx 10^{5}$.

- LSBA is the weight of the least significant bit of the accumulator. For example, if LSBA = -50 , the accumulator can hold data accurate to $2^{-50} \approx 10^{-15}$.

The accumulator width $w_{a}$ is then computed as MSBA - LSBA +1 , for instance 68 bits in the previous example. Here, 68 bits represents a wide range and high accuracy, and still additions on this format will have one-cycle latency for practical frequencies on recent FPGAs. If this is not enough, the frequency can be improved thanks to partial carry save [13], but this was not useful in the present work. For comparison, for the same frequency, a floating-point adder has a latency of 7 to 10 cycles, depending on the target, with an initiation interval of about 4 cycles.

3.3.2 Implementation within an HLS Tool. This accumulator has been implemented in $\mathrm{C} / \mathrm{C}++$, using arbitrary-precision fixed point types (ap_int) and the Modern Arithmetic Tools for HLS library [44].

For modularity purposes, FloatToFix and FixToFloat are wrapped into $\mathrm{C} / \mathrm{C}++$ functions (33 and 28 lines of code, respectively). Their calls are inlined to enable HLS optimizations. The implementation of the FloatToFix function has one more parameter, called MaxMSBX: it defines the largest possible exponent of the floating-point input. Its default value is equal to MSBA, but it may be smaller, when the application context dictates an upper bound on the magnitude of the input to FloatToFix. In this case, the size of the shifter (Figure 5) can be reduced.

Because the internal accumulation is performed on a fixed-point integer representation, the combinational delay between two accumulations is lower compared to a full floating-point addition. HLS tools can take advantage of this delay reduction by more aggressive loop pipelining (with a shorter initiation interval), resulting in a design with a shorter overall latency.

3.3.3 Validation. To evaluate and refine this implementation, we used Listing 10, which we compared to Listings 8 and 9.

The parameters chosen for the accumulator in this experiment are the following:

- $\mathrm{MSBA}=17$. Indeed, as we are adding $\cos (i) 100 \mathrm{~K}$ times, an upper bound is $100 \mathrm{~K}$, which can be encoded in 17 bits.

- $\operatorname{MaxMSBX}=1$ as the maximum input value is 1 .

- $\mathrm{LSBA}=-50$ : The accumulator itself will be accurate to the 50th fractional bit. Note that a float input will see its mantissa rounded by FloatToFix only if its exponent is smaller than $2^{-25}$, which is very rare. In other words, this accumulator is much more accurate than the data that is thrown to it.

The results are reported in the two rightmost columns of Table 6. The Accuracy line of the table reports the number of correct bits of each implementation, after the result has been converted to a 


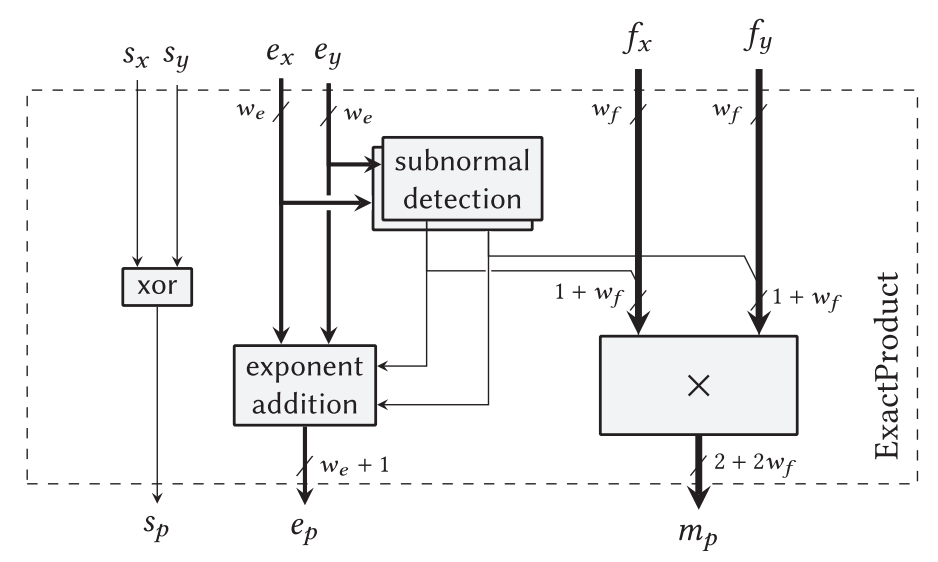

Fig. 7. Exact floating-point multiplier.

float. The proposed fixed-point accumulator achieves minimal latency and maximum accuracy, for a LUT usage comparable to the usual float accumulation of Listing 8.

Removing the control part from Listing 10 reduces resource usage to 395 LUTs, which is very comparable to the 375 LUTs of the corresponding FloPoCo-generated VHDL (which does not include this control nor subnormal support). This shows that there is no overhead due to the use of HLS.

Using this implementation method, we also created an exact floating-point multiplier with the final rounding removed [13]. This component is depicted in Figure 7. The corresponding function is called ExactProduct and represents 44 lines of code. The result mantissa is twice as large as the input mantissas (48 bits in single precision). To add it to the large accumulator, the Float-to-Fix block has to be adapted: in the sequel, it is called ExactProductFloatToFix (21 lines of code).

\subsection{The Compiler Side: Source-to-Source Transformation}

The previous section and previous work by various groups [20, 40] have shown that Vivado HLS can be used to synthesize very efficient specialized floating-point operators that rival in quality with those generated by FloPoCo or vendor tools. Our goal is now to study how such optimizations can be automated. More precisely, we aim at automatically optimizing Listing 8 into Listing 10 and generalizing this transformation to many more situations.

For convenience, this optimization was also developed as a source-to-source transformation implemented within GeCoS and is publicly available (https://gitlab.inria.fr/gecos/gecos-arith).

This part focuses on two computational patterns: the accumulation and the sum of product. Both are specific instances of the reduction pattern, which can be optimized by many compilers or parallel runtime environments. Reduction patterns are exposed to the compiler/runtime either though user directives (e.g., \#pragma reduce in openMP) or automatically inferred using static analysis techniques [16, 38].

As the problem of detecting reductions is not the main focus on this work, our tool uses a straightforward solution to the problem using a combination of a user-provided compiler directive (pragma) and some simple program analysis.

More specifically, the proposed code transformation is triggered by a pragma that defines a target accumulation variable, along with application-level information such as the dynamic range of the accumulated data or the overall target accuracy. 


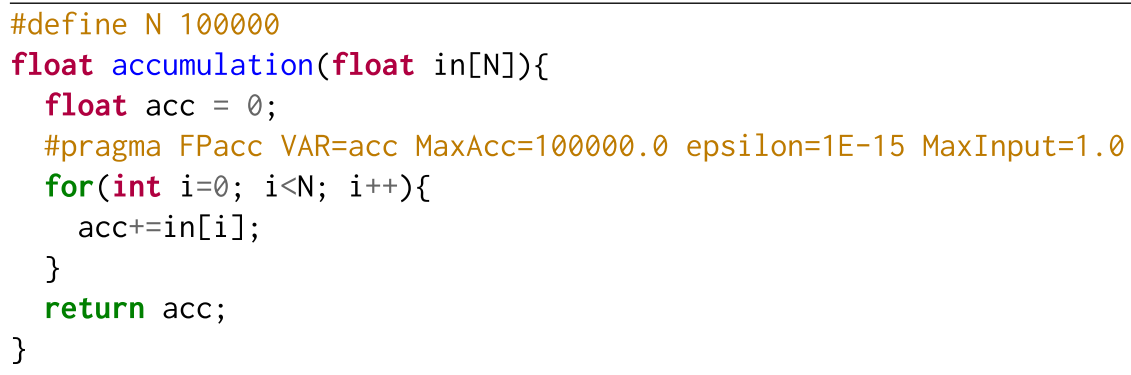

Listing 11. Illustration of the use of a pragma for the usual accumulation.

We found this approach easier, more general, and less invasive than those attempting to convert a whole floating-point program into a fixed-point implementation [39].

The pragma approach has another advantage: we advocate relaxing the standard $\mathrm{C}$ semantics, which is dangerous in principle. Therefore, the user should explicitly ask for it. A pragma may allow such relaxation in a local, controlled way, without sacrificing standard compliance for the rest of the program.

3.4.1 Proposed Compiler Directive. In imperative languages such as C, reductions are implemented using for or while loop constructs. The proposed compiler directive must therefore appear right outside such a construct. Listing 11 illustrates its usage on the code of Listing 8 .

The pragma must contain the following information:

- The keyword FPacc, which triggers the transformation.

- The name of the variable in which the accumulation is performed, preceded with the keyword VAR. In the example, the accumulation variable is acc.

In addition, it may provide the following information:

- The maximum value that can be reached by the accumulator through the use of the MaxAcc keyword. This value is used to determine MSBA.

- The desired accuracy of the accumulator using the epsilon keyword. This value is used to determine LSBA.

- The maximum value that the inputs can take, using the MaxInput keyword. This value is used to determine MaxMSBX. If this information is not provided, then MaxMSBX is set to MSBA.

Note that the user can quietly overestimate the maximum value of the accumulator without major impact on area. For instance, overestimating MaxAcc by a factor of 10 only adds 3 bits to the accumulator width.

In cases when the user is unable to provide such information, a full-range Kulisch accumulator is used as a fall-back strategy-a recent study has evaluated different implementations of the full Kulisch accumulator in an FPGA context [42].

3.4.2 Proposed Code Transformation. The proposed transformation operates on the compiler program intermediate representation (IR). It relies on the ability to identify loops constructs and expose def/use relations between instructions within a basic block in the form of an operation dataflow graph (DFG).

To illustrate the transformation, consider the non-trivial code of Listing 12 . This program performs a reduction into the variable sum, involving both sums and sums of product operations. 


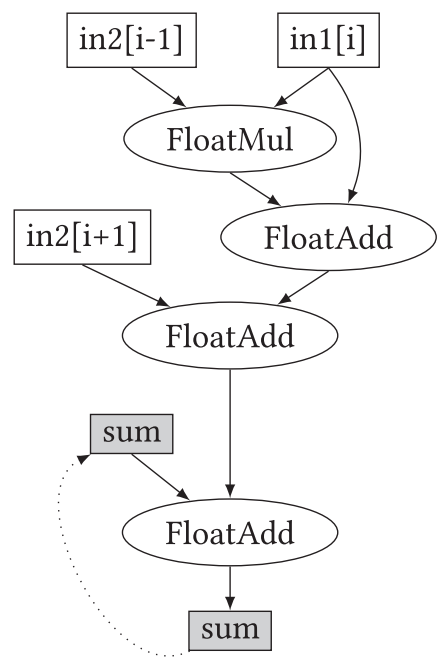

(a) Loop body DFG

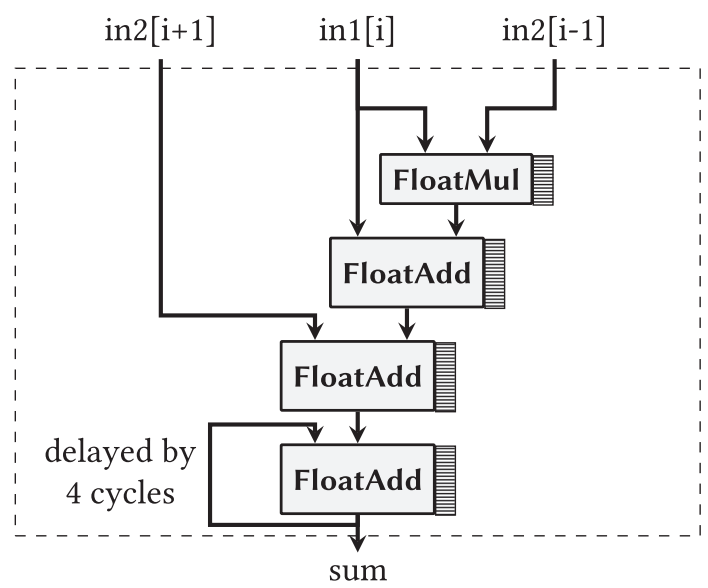

(b) Architecture

Fig. 8. DFG of the loop body from Figure 12 (left) and its corresponding architecture (right). FloatMul and FloatAdd correspond to floating-point multipliers and adders, respectively.

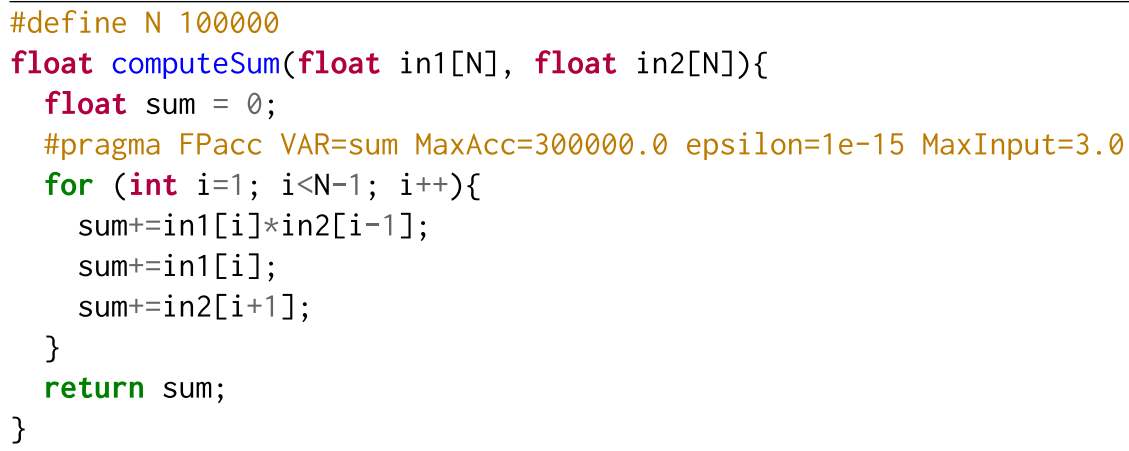

Listing 12. Simple reduction with multiple accumulation statements.

Figure 8(a) shows the operation DFG for the loop body of this program. In this figure, dotted arrows represent loop-carried dependencies between operations belonging to distinct loop iterations. Such loop-carried dependencies have a very negative impact on the kernel latency, as they prevent loop pipelining. For example, when using a pipelined floating-point adder with an inner latency of four cycles, the HLS tool will schedule a new iteration of the loop at best every four cycles.

As illustrated in Figure 9(a), the proposed transformation hoists the floating-point normalization step out of the loop and performs the accumulation using fixed-point arithmetic. Since integer add operations can be implemented with a one-cycle delay at our target frequency, the HLS tool may now be able to initiate a new iteration every cycle, improving the overall latency by a factor of 4 .

The code transformation first identifies all relevant basic blocks (i.e., those associated to the pragma directive). It then performs a backward traversal of the DFG, starting from a FloatAdd node that writes to the accumulation variable identified by the \#pragma. 


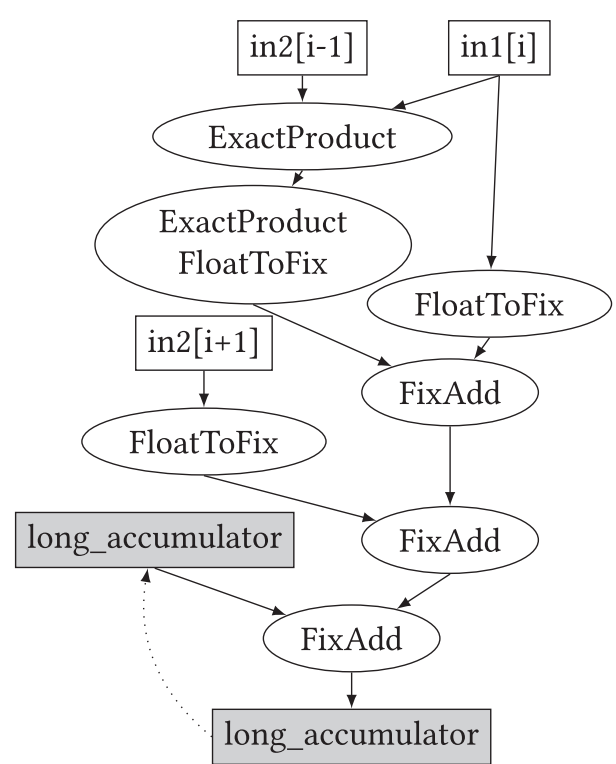

(a) Loop body DFG

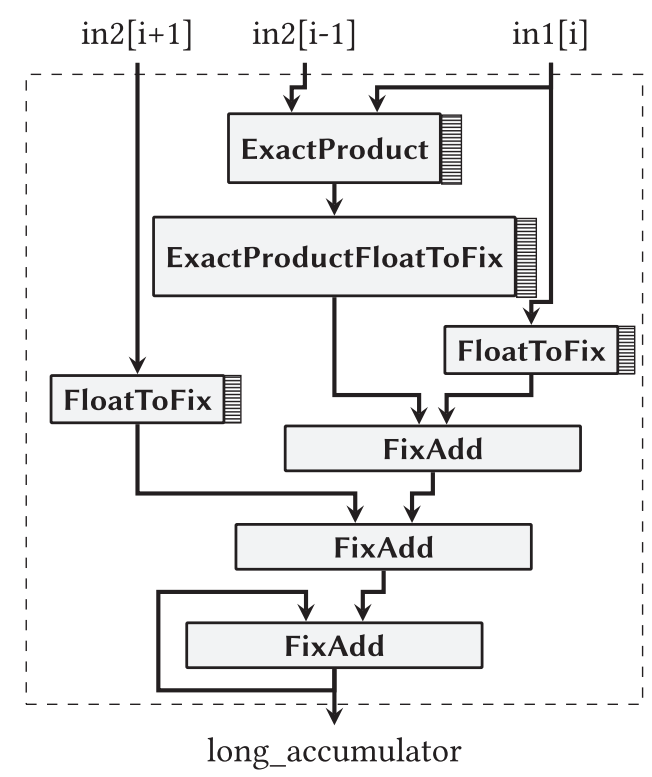

(b) Architecture

Fig. 9. DFG of the loop body from Figure 12 (left) and its corresponding architecture (right) after transformations.

During this traversal, the following actions are performed depending on the visited nodes:

- A node with the summation variable is ignored.

- A FloatAdd node is transformed to an accurate fixed-point adder. The analysis is then recursively launched on that node.

- A FloatMul node is replaced with a call to the ExactProduct function followed by a call to ExactProdFloatToFix.

- Any other node has a call to FloatToFix inserted.

This algorithm transforms the DFG from Figure 8(a) into the new DFG shown on Figure 9(a). In addition, a new basic block containing a call to FixToFloat is inserted immediately after the transformed loop to expose the floating-point representation of the results to the remainder of the program.

From there, it is then possible to regenerate the corresponding $C$ code. As an illustration of the whole process, Figures 8(b) and 9(b) describe the architectures corresponding to the code before and after the transformation.

3.4.3 Evaluation on the Code of Listing 12. The proposed transformations work on non-trivial examples such as the one represented in Listing 12. Table 7 shows how resource consumption depends on epsilon, and all other parameters being those given in the pragma of Listing 12 .

Compared to the classical IEEE-754 implementation, the transformed code uses similar or more LUTs depending on the precision of the accumulator. The original code requires 3 DSPs for the floating-point multiplier (the adder is implemented in LUTs). The transformed code only requires 2 DSPs as the floating-point multiplier IP is removed for the custom exact multiplier that removes the normalization part (which required an extra DSP). In all cases, on this example, the transformed code has its latency reduced by a factor equal to 9. 
Table 7. Comparison between the Usual Code from Listing 12 and Its Transformed Equivalent

\begin{tabular}{ccccc}
\hline & Usual & $\begin{array}{c}\text { Transformed } \\
\text { LSBA }=-14\end{array}$ & $\begin{array}{c}\text { Transformed } \\
\text { LSBA }=-20\end{array}$ & $\begin{array}{c}\text { Transformed } \\
\text { LSBA }=-50\end{array}$ \\
\cline { 2 - 5 } LUTs & 534 & 501 & 587 & 1089 \\
DSPs & 3 & 2 & 2 & 2 \\
Latency & $900 \mathrm{~K}$ & $100 \mathrm{~K}$ & $100 \mathrm{~K}$ & $100 \mathrm{~K}$ \\
\hline
\end{tabular}

Results obtained with Vivado HLS 2019.1 targeting Kintex7. All of these versions run at $100 \mathrm{MHz}$.

\subsection{Evaluation on the EEMBC FPMark Benchmarks}

To evaluate the relevance of the proposed transformations on real-life programs, we used the EEMBC FPMark benchmark suite [17]. The only transformations considered in this evaluation are the ones presented in Section 3.4. This suite consists of 10 programs. A first result is that half of these programs contain floating-point accumulations:

- Enhanced Livermore Loops (1/16 kernels contains a sum of product).

- LU Decomposition (multiple accumulations).

- Neural Net (multiple sum of products).

- Fourier Coefficients (one accumulation).

- Black Scholes (one accumulation).

The following focuses on these and ignores the other half (Fast Fourier Transform, Horner's method, Linpack, ArcTan, Ray Tracer), as they do not benefit from the transformations (code is unchanged).

Most benchmarks come in both single- and double-precision versions. We focus here on the single-precision benchmarks. Double-precision benchmarks lead to the same conclusions.

3.5.1 Benchmarks and Accuracy: Methodology. Each benchmark comes with a golden reference against which the computed results are compared. As the proposed transformations are controlled by the accuracy, it may happen that the transformed benchmark is less accurate than the original. In this case, it will not pass the benchmark verification test, and rightly so.

A problem is that the transformed code will also fail the test if it is more accurate than the original. Indeed, the golden reference is the result of a certain combination of rounding errors using the standard FP formats, which we do not attempt to replicate.

To work around this problem, each benchmark was first transformed into a high-precision version where the accumulation variable is a 10,000-bit floating-point number using the MPFR library [21]. We used the result of this highly accurate version as a "platinum" reference, against which we could measure the accuracy of the benchmark's golden reference. This allowed us to choose our epsilon parameter such that the transformed code would be at least as accurate as the golden reference. This way, the epsilon of the following results is obtained through profiling. The accuracy of the obtained results are computed as the number of correct bits of the result.

Let us review in detail how each benchmark is improved by the proposed transformation. All synthesis results (before and after transformation) are given in Table 8.

Enhanced Livermore Loops. This program contains 16 kernels of loops that compute numerical equations. Among these kernels, there is one that performs a sum of product (banded linear equations). This kernel computes the sum of 20,000 products 300 times, resulting in $6 \mathrm{M}$ accumulated 
Table 8. Synthesis Results of Benchmarks Before and After Sum-of-Product Transformations

\begin{tabular}{|c|c|c|c|c|c|c|}
\hline Benchmark & Type & LUTs & DSPs & BRAM & Latency & Accuracy \\
\hline \multirow{2}{*}{ Livermore } & Original & 398 & 5 & 0 & $54 \mathrm{M}$ & 11 bits \\
\hline & Transformed & 608 & 4 & 0 & $6 \mathrm{M}$ & $13 \mathrm{bi}$ \\
\hline \multirow{2}{*}{ LU-8 } & Original & 435 & 3 & 0 & 38 & $8-23$ bi \\
\hline & Transformed & 274 & 2 & 0 & 14 & $23 \mathrm{~b}$ \\
\hline \multirow{2}{*}{ LU-45 } & Original & 472 & 4 & 0 & 186 & $8-23 \mathrm{bi}$ \\
\hline & Transformed & 300 & 3 & 0 & 51 & $23 \mathrm{bi}$ \\
\hline \multirow{3}{*}{ Black Scholes } & Original & 14388 & 147 & 0 & $4 \mathrm{M}$ & $19 \mathrm{bi}$ \\
\hline & Transformed II=4 & 13623 & 138 & 0 & $4 \mathrm{M}$ & $23 \mathrm{bi}$ \\
\hline & Transformed II=1 & 22646 & 308 & 0 & $1 \mathrm{M}$ & $23 \mathrm{bi}$ \\
\hline \multirow{2}{*}{ Fourier Coefficients } & Original & 22707 & 319 & 14 & $34 \mathrm{~K}^{*}$ & $6 \mathrm{bi}$ \\
\hline & Transformed & 13990 & 145 & 7 & $25 \mathrm{~K}^{*}$ & $11 \mathrm{bi}$ \\
\hline
\end{tabular}

Results obtained with Vivado HLS 2019.1 targeting Kintex7. All of these versions run at $100 \mathrm{MHz}$.

${ }^{*}$ Obtained through cosimulation.

terms. The values accumulated are pre-computed. This is a perfect candidate for the proposed transformations.

For this benchmark, the optimal accumulation parameters were found to be the following:

MaxAcc $=50000.0$ epsilon $=1$ e-5 MaxInput $=22000.0$.

As previously, latency is vastly improved while achieving improved accuracy. The area is comparable, as the operator implemented in the transformation uses a bit more LUTs and fewer DSPs (as discussed in Section 3.4.3).

LU Decomposition and Neural Net. Both the LU Decomposition and Neural Net programs contain multiple nested small accumulations. In the LU Decomposition program, an inner loop accumulates between 8 and 45 values. Such accumulations are performed more than $7 \mathrm{M}$ times. In the Neural Net program, inner loops accumulate between 8 and 45 values, and such accumulations are performed more than $5 \mathrm{~K}$ times.

Both programs accumulate values from registers or memory that are already computed. It makes these programs good candidates for the proposed transformations.

Vivado HLS is unable to predict a latency for these designs due to the non-constant loop trip counts. As a consequence, instead of presenting results for the complete benchmark, we restrict ourselves to the LU innermost loops. Table 8 shows the results obtained for the smallest (8 terms) and the largest (45 terms) sums of products in lines LU-8 and LU-45, respectively. The latency is vastly improved even for the smallest one. The accuracy results of the original code here varies from 8 to 23 bits between different instances of the loops. To have a fair comparison, we generated a conservative design that performs 23-bit accuracy on all loops, using a sub-optimal amount of resources. Still, the transformed code requires fewer resources.

Black Scholes. This program contains an accumulation that sums 200 terms. These terms are computed performing polynomial approximations and exponentiations from the input data. The result of the accumulation is divided by a constant (that could be further optimized by using transformations from Section 2). This process is performed 5,000 times, and hence it results in $1 \mathrm{M}$ accumulations.

Here the optimal accumulator parameters are the following:

$$
\text { MaxAcc }=245000.0 \text { epsilon=1e-4 MaxInput }=278.0 \text {. }
$$


This gives us an accumulator that uses 19 bits for the integer part and 10 bits for the fractional part.

The original code is only able to achieve an initiation interval of four cycles (limited by the accumulation). Because of this four-cycle latency between two iterations, Vivado HLS is able to reuse some of the hardware that computes the terms to accumulate in order to reduce the hardware consumption. For example, a floating-point exponential IP is reused for the computation of a single term to accumulate.

The transformed code is able to achieve an initiation interval of one cycle. Therefore, this exponential hardware can no longer be reused and must be replicated to feed the accumulator. This explains the increased resource usage presented as Transformed II $=1$.

As the transformed code can achieve a one-cycle initiation interval, it is also able to achieve any higher initiation intervals (two, three, or four cycles), maintaining the same accuracy. The transformation then offers a trade-off between latency and resource usage. The most conservative alternative (keeping the original latency but for reduced resource usage and improved accuracy) is presented as Transformed $I I=4$.

Fourier Coefficients. This program computes the coefficients of a Fourier series, using an accumulation performed in single precision. It comes in three different configurations: small, medium, and big. Each of them computes the same algorithm but with a different number of iterations. The big version is supposed to compute the most accurate answer. Therefore, the parameters of the custom accumulator are chosen according to this version:

$$
\text { MaxAcc }=6000.0 \text { epsilon=1e-7 MaxInput }=10.0 \text {. }
$$

This results in an accumulator using 14 bits for the integer part and 24 bits for the fractional part.

Table 8 shows that area is considerably reduced, whereas accuracy is improved by 5 bits (more than one order of magnitude). However, Vivado HLS cannot statically compute the overall latency due to the use of a floating-point power function. Therefore, the latencies reported here are obtained through cosimulation. To keep the cosimulation duration reasonable, only the small dataset is used. It computes 100 accumulations of 20 terms, followed by other computations. In that case, the latency is also reduced.

\section{CONCLUSION}

This study first demonstrates how today's commercial HLS tools, such as Vivado HLS and Intel HLS, fail at exploiting full FPGA potential when dealing with numerical programs, particularly with floating-point numbers.

One reason is the historical heritage of processor-oriented compiler back-ends. We advocate the integration in HLS compilers of well-known hardware-oriented, low-level arithmetic optimizations. Some of them can be applied at the level of the front-end, as showcased by the sourceto-source approach used in this study. Others need to be integrated as optimization passes on a compiler IR. The benefits demonstrated by this study, both in terms of resource usage and latency, make these optimizations a must do.

This study also looks a bit further toward compiler directives that would instruct the compiler to treat floats as reals in a local, user-controlled way. The role of the compiler is then to achieve the prescribed accuracy (defined with respect to an ideal, exact computation on the reals) with the best performance at minimal cost. In the context of hardware synthesis, this approach presents the opportunity to use non-standard internal formats and operators, as demonstrated in the case study of floating-point sums and sums of products. With improvements in performance, cost, and accuracy, this is a promising research direction for HLS tools. 


\section{REFERENCES}

[1] IEEE. (n.d.). IEEE Standard for Binary Floating-Point Arithmetic. Standard 754-1985. IEEE, Los Alamitos, CA.

[2] Intel. 2019. Intel High Level Synthesis Compiler: Best Practices Guide. Intel.

[3] Xilinx. 2019. Vivado Design Suite User Guide: High-Level Synthesis. Xilinx.

[4] Levent Aksoy, Eduardo Costa, Paulo Flores, and Jose Monteiro. 2007. Optimization of area in digital FIR filters using gate-level metrics. In Proceedings of the Design Automation Conference. IEEE, Los Alamitos, CA, 420-423.

[5] Randy Allen and Ken Kennedy. 2002. Optimizing Compilers for Modern Architectures. Morgan Kaufmann.

[6] Nicolas Brisebarre, Florent de Dinechin, and Jean-Michel Muller. 2008. Integer and floating-point constant multipliers for FPGAs. In Proceedings of the 2008 International Conference on Application-Specific Systems, Architectures, and Processors. IEEE, Los Alamitos, CA, 239-244.

[7] Gabriel Caffarena, Juan A. Lopez, Carlos Carreras, and Octavio Nieto-Taladriz. 2006. High-level synthesis of multiple word-length DSP algorithms using heterogeneous-resource FPGAs. In Proceedings of the International Conference on Field Programmable Logic and Applications. IEEE, Los Alamitos, CA.

[8] Ken Chapman. 1993. Fast integer multipliers fit in FPGAs (EDN 1993 design idea winner). EDN Magazine 10 (May 1993).

[9] Jason Cong, Muhuan Huang, Peichen Pan, Yuxin Wang, and Peng Zhang. 2016. Source-to-source optimization for HLS. In FPGAs for Software Programmers. Springer, 137-163.

[10] Florent de Dinechin. 2012. Multiplication by rational constants. IEEE Transactions on Circuits and Systems II: Express Briefs 52, 2 (2012), 98-102.

[11] Florent de Dinechin, Silviu-Ioan Filip, Luc Forget, and Martin Kumm. 2019. Table-based versus shift-and-add constant multipliers for FPGAs. In Proceedings of the 26th IEEE Symposium on Computer Arithmetic. IEEE, Los Alamitos, CA.

[12] Florent de Dinechin and Bogdan Pasca. 2013. Reconfigurable arithmetic for high performance computing. In HighPerformance Computing Using FPGAs. Springer, 631-664.

[13] Florent de Dinechin, Bogdan Pasca, Octavian Cret, and Radu Tudoran. 2008. An FPGA-specific approach to floatingpoint accumulation and sum-of-products. In Proceedings of the International Conference on Field-Programmable Technology. IEEE, Los Alamitos, CA, 33-40.

[14] Andrew G. Dempster and Malcolm D. Macleod. 1994. Constant integer multiplication using minimum adders. IEE Proceedings-Circuits, Devices and Systems 141, 5 (1994), 407-413.

[15] Michael Dibrino. 2005. Floating point multiplier/accumulator with reduced latency and method thereof. US Patent $6,904,446$.

[16] Johannes Doerfert, Kevin Streit, Sebastian Hack, and Zino Benaissa. 2015. Polly's polyhedral scheduling in the presence of reductions. In Proceedings of the International Workshop on Polyhedral Compilation Techniques.

[17] EEMBC, the Embedded Microprocessor Benchmark Consortium. 2013. Introduction to the EEMBC FPMark ${ }^{\mathrm{TM}}$ FPMark Floating-Point Benchmark Suite. Retrieved January 29, 2020 from http://www.eembc.org/fpmark.

[18] Bruce M. Fleischer, Juergen Haess, Michael Kroener, Martin S. Schmookler, Eric M. Schwarz, and Son Dao-Trong. 2010. System and method for a floating point unit with feedback prior to normalization and rounding. US Patent 7,730,117.

[19] Antoine Floc'h, Tomofumi Yuki, Ali El-Moussawi, Antoine Morvan, Kevin Martin, Maxime Naullet, Mythri Alle, et al. 2013. GeCoS: A framework for prototyping custom hardware design flows. In Proceedings of the International Working Conference on Source Code Analysis and Manipulation. IEEE, Los Alamitos, CA, 100-105.

[20] Luc Forget, Yohann Uguen, Florent de Dinechin, and David Thomas. 2019. A type-safe arbitrary precision arithmetic portability layer for HLS tools. In Proceedings of the International Symposium on Highly Efficient Accelerators and Reconfigurable Technologies (HEART'19).

[21] Laurent Fousse, Guillaume Hanrot, Vincent Lefèvre, Patrick Pélissier, and Paul Zimmermann. 2007. MPFR: A multiple-precision binary floating-point library with correct rounding. ACM Transactions on Mathematical Software 33, 2 (2007), 1-14.

[22] Marcel Gort and Jason H. Anderson. 2013. Range and bitmask analysis for hardware optimization in high-level synthesis. In Proceedings of the Asia and South Pacific Design Automation Conference. IEEE, Los Alamitos, CA, 773-779.

[23] Oscar Gustafsson. 2007. Lower bounds for constant multiplication problems. ACM Transactions on Circuits and Systems II: Express Briefs 54, 11 (Nov. 2007), 974-978.

[24] James Hrica. 2012. Floating-Point Design with Vivado HLS. Application Note. Xilinx.

[25] Qijing Huang, Ruolong Lian, Andrew Canis, Jongsok Choi, Ryan Xi, Nazanin Calagar, Stephen Brown, and Jason Anderson. 2015. The effect of compiler optimizations on high-level synthesis-generated hardware. ACM Transactions on Reconfigurable Technology and Systems 8, 3 (2015), 14.

[26] ISO. 2011. C11 Standard. ISO/IEC 9899:2011. ISO.

[27] Edin Kadric, Paul Gurniak, and André DeHon. 2016. Accurate parallel floating-point accumulation. IEEE Transactions on Computers 65, 11 (2016), 3224-3238. 
[28] Nachiket Kapre and André DeHon. 2007. Optimistic parallelization of floating-point accumulation. In Proceedings of the Symposium on Computer Arithmetic. IEEE, Los Alamitos, CA, 205-216.

[29] Ulrich Kulisch and Van Snyder. 2011. The exact dot product as basic tool for long interval arithmetic. Computing 91, 3 (2011), 307-313.

[30] Martin Kumm, Oscar Gustafsson, Mario Garrido, and Peter Zipf. 2018. Optimal single constant multiplication using ternary adders. IEEE Transactions on Circuits and Systems II: Express Briefs 65, 7 (2018), 928-932.

[31] Zhen Luo and Margaret Martonosi. 2000. Accelerating pipelined integer and floating-point accumulations in configurable hardware with delayed addition techniques. IEEE Transactions on Computers 49, 3 (2000), 208-218.

[32] Robert Morgan. 1998. Building an Optimizing Compiler. Digital Press.

[33] Steven Muchnick. 1997. Advanced Compiler Design Implementation. Morgan Kaufmann.

[34] Jean-Michel Muller, Nicolas Brunie, Florent de Dinechin, Claude-Pierre Jeannerod, Mioara Joldes, Vincent Lefèvre, Guillaume Melquiond, Nathalie Revol, and Serge Torres. 2018. Handbook of Floating-Point Arithmetic (2nd ed.). Birkhauser, Boston, MA.

[35] Razvan Nane, Vlad-Mihai Sima, Christian Pilato, Jongsok Choi, Blair Fort, Andrew Canis, Yu Ting Chen, et al. 2015. A survey and evaluation of FPGA high-level synthesis tools. IEEE Transactions on Computer-Aided Design of Integrated Circuits and Systems 35, 10 (2015), 1591-1604.

[36] Bogdan Pasca. 2012. Correctly rounded floating-point division for DSP-enabled FPGAs. In Proceedings of the International Conference on Field Programmable Logic and Applications. IEEE, Los Alamitos, CA, 249-254.

[37] Louis-Noël Pouchet. 2012. Polybench: The Polyhedral Benchmark Suite. Retrieved March 25, 2019 from https://dl. acm.org/doi/abs/10.1145/1240233.1240234.

[38] Xavier Redon and Paul Feautrier. 2000. Detection of scans. Parallel Algorithms and Applictations 15, 3-4 (2000), 229263.

[39] Olivier Sentieys, Daniel Menard, David Novo, and Karthick Parashar. 2014. Automatic fixed-point conversion: A gateway to high-level power optimization. Tutorial presented at the IEEE/ACM Design Automation and Test in Europe Conference.

[40] David Thomas. 2019. Templatised soft floating-point for high-level synthesis. In Proceedings of the 27th International Symposium on Field-Programmable Custom Computing Machines. IEEE, Los Alamitos, CA.

[41] Jason Thong and Nicola Nicolici. 2011. An optimal and practical approach to single constant multiplication. IEEE Transactions on Computer-Aided Design of Integrated Circuits and Systems 30, 9 (2011), 1373-1386.

[42] Yohann Uguen and Florent de Dinechin. 2017. Design-space exploration for the Kulisch accumulator. CCSD HAL. Retrieved January 29, 2020 from https://hal.archives-ouvertes.fr/hal-01488916v2.

[43] Yohann Uguen, Florent de Dinechin, and Steven Derrien. 2017. Bridging high-level synthesis and application-specific arithmetic: The case study of floating-point summations. In Proceedings of the 27th International Conference on Field Programmable Logic and Applications. IEEE, Los Alamitos, CA.

[44] Yohann Uguen, Luc Forget, and Florent de Dinechin. 2019. Evaluating the hardware cost of the posit number system. In Proceedings of the 29th International Conference on Field-Programmable Logic and Applications.

[45] H. Fatih Ugurdag, Florent de Dinechin, Y. Serhan Gener, Sezer Gören, and Laurent-Stéphane Didier. 2017. Hardware division by small integer constants. IEEE Transactions on Computers 66, 12 (2017), 2097-2110.

[46] Wim Vanderbauwhede and Khaled Benkrid. 2013. High-Performance Computing Using FPGAs. Vol. 3. Springer.

[47] Yevgen Voronenko and Markus Püschel. 2007. Multiplierless multiple constant multiplication. ACM Transactions on Algorithms 3, 2, Article 11 (2007). https://dl.acm.org/doi/abs/10.1145/1240233.1240234

[48] E. George Walters. 2017. Reduced-area constant-coefficient and multiple-constant multipliers for Xilinx FPGAs with 6-Input LUTs. Electronics 6, 4 (2017), 101.

[49] Michael J. Wirthlin. 2004. Constant coefficient multiplication using look-up tables. fournal of VLSI Signal Processing Systems for Signal, Image and Video Technology 36, 1 (2004), 7-15.

Received July 2019; revised December 2019; accepted December 2019 